\title{
SOHO: A PRISMA PRECURSOR
}

\section{P. LEMAIRE}

I.A.S., Bat.121, Université de Paris XI, 91405 Orsay Cedex

\begin{abstract}
Probing the interior of the Sun, a slow rotator G2 V main sequence star, and connecting the atmospheric structure to inside phenomena is one of the objectives of the SOHO mission. An overview of the helioseismology and of the coronal instruments is given. It furnishes a guide line for missions that are dedicated to the probing of stellar interiors in using microvariability and activity as tracers, such as the PRISMA study.
\end{abstract}

\section{INTRODUCTION}

SOHO (SOlar and Heliospheric Observatory) is an ESA/NASA solar mission to be launched in 1995 at the $\mathrm{L}_{1}$ Sun-Earth Lagrangian position. Continuous observations will be performed during more than 2 years.

The mission objectives are:

- to infer the internal solar structure through helioseismology sounding technique. Continuous observations of the global doppler velocity oscillation and the global irradiance variation will provide informations on the deep core from oscillation modes $l=0,1$ and 2. Coarse resolution on the solar disc in irradiance gives access to intermediate levels. High resolution on the solar disc in doppler velocity is a probe of the upper part of the internal structure where the convection shapes and permanently disturbs the structuration.

- to observe the outer part of the solar atmosphere and provide criteria in order to infer coronal heating mechanisms and processes that impulse and drive the solar wind. Combining the solar global view from the chromosphere to the corona with detailed analysis of resolved structures will constrain any theoretical consideration. Observations on and outside the solar disc will draw the complete (three dimensions) outline of the coronal structuration.

- to make in-situ measurement of the interplanetary plasma.

\section{HELIOSEISMOLOGY SEGMENT}

Global doppler velocity measurement (GOLF- Gabriel et al, 1989) $o$ use of a sodium resonance cell to provide accurate absolute wavelength reference 
o full Sun doppler velocity and global magnetic field measurements

o expected velocity accuracy: $1 \mathrm{~mm} / \mathrm{s}$ in the $10^{-7}-6 \cdot 10^{-3} \mathrm{~Hz}$ frequency range

o oscillation modes $\mathrm{l}=0$ - 4

o continuous observations during 2 years

Solar Oscillation Imager (SOI - Scherrer et al, 1989)

o Michelson Doppler Imager instrument

o full solar image analysis: line of sight velocity, transverse velocity, line and continuum intensity, and detailed magnetic field

02 and 0.7 arcsec pixels (1920 arcsec solar diameter)

o line of sight velocity sensitivity: $<1 \mathrm{~m} / \mathrm{s}$

o oscillation modes up to $l=1000$

o continuous observation 2 months/year (partial otherwise)

Solar monitor experiment (VIRGO - Fröhlich et al, 1989))

0 active cavity radiometers, photometers and low resolution imager

o frequencies, amplitude and phases of intensity oscillations mode in the frequency range $10^{-7}-10 \mathrm{mHz}$

o sensitivity better than $10^{-7}$ magnitude

o oscillation modes $1=0-7$

o continuous observations during 2 years

Combining the helioseismology segment provides:

o high accuracy determination of modes in the range $1=0$ to 1000 measured in doppler velocity

o high accuracy determination of modes in the range $1=0$ to 7 measured as brightness variations. Comparison between velocity and brightness oscillations gives further constraints on the generation and the propagation of oscillations.

o a full set of measurements to derive internal structure and dynamics of the solar interior.

\section{SOLAR ATMOSPHERIC SEGMENT}

Eull disc Extreme ultraviolet Imager (EIT - Delaboudiniere et al, 1989)

o Multi-wavelength imager at 17.1, 19.5, 28.4 and $304 \mathrm{~nm}$ in upper chromosphere and coronal lines

o 3 arcsec pixels

o full solar disc field-of-view

Coronal Diagnostic Spectrometer (CDS - Patchett et al, 1989)

o grazing incidence telescope with a grazing incidence spectrometer ( 15 - 80 $\mathrm{nm}$ spectral range) and a normal incidence spectrometer ( 2 bands in $24-80 \mathrm{~nm}$ range)

o resolving power $2000-9000(\lambda / \Delta \lambda)$

0 angular resolution $\sim 2$ arcsec

o capability of small field scanning ( $240 \times 240 \mathrm{arcsec}$ ) and wide field (up to the full solar disc and .5 solar radius outside)

o diagnostic of spectral lines in the $10^{4}$. few $10^{6} \mathrm{~K}$ range 
Ultra violet spectrometer (SUMER - Wilhelm et al, 1989)

o normal incidence telescope and spectrometer in the $50-160 \mathrm{~nm}$ spectral range o resolving power: $20000-40000(\lambda \Delta \lambda)$

0 angular resolution $\sim 1$ arcsec

o capability of small field scanning $(300 \times 300$ arcsec) and large field scanning (full solar disc and .5 solar radius outside)

o detailed profile of spectral lines in the $10^{4}-10^{6} \mathrm{~K}$ temperature range

UltraViolet Coronograph (UVCS - Kohl et al, 1989)

o scanning normal incidence coronograph from 0.2 to 9 solar radii above the limb with spectrometric observations in the 49.5- 61. nm and 102.- $125 . \mathrm{nm}$ wavelength bands.

o resolving power $500-9000(\lambda / \Delta \lambda)$

0 angular resolution from 10 arcsec to arcmin

o diagnostic of density, velocity and temperature

White light coronograph (LASCO - Michels,Brueckner et al, 1989)

o 3 complementary coronographs extending the measurements within fields of $0.1-2.0$ (C1), $0.5-5.0(\mathrm{C} 2), 2.0-29.0$ (C3) solar radii above the solar limb o angular resolution (pixel size) 5.6" (C1), 11.4" (C2) and 56" (C3) o measurements of coronal structure and evolution.

Combining coronal segment provides:

o a global and detailed view of the corona

o long term and short term evolutions of coronal structures

o constraints on heating mechanisms and acceleration of the solar wind

o rotational rate at several heights above the limb

$o$ access to detailed processes acting in the small scale structures in quiet and active solar area

\section{CONCLUSION}

Combining the heliosismology and atmospheric segments, SOHO will provide: o a complete sounding of the Sun from the deep core to the most external layers o a unique tool to test stellar theories at a given age, chemical composition, mass and luminosity

o a set of fine diagnostics on small area of the Sun and on the full Sun

\section{REFERENCES}

THE SOHO MISSION, Scientific and technical aspects of the instruments, ed.V. Domingo, ESA SP-1104, 1989 